\section{E-Zigaretten schädigen junge Lungen}

McConnell R et al. Electronic-cigarette Use and Respiratory Symptoms in Adolescents. Am J Respir Crit Care Med 2017; 195: 1043 - 1049

In den USA rauchten 2015 bereits $15 \%$ der Heranwachsenden im 9. bis 12. Schuljahrgang E-Zigaretten und ähnliche Produkte - Tendenz steigend. Auch die Zahl der E-Rauchprodukte und der Aromen steigt immer weiter an. Geruchsstoffe, flüchtige Aldehyde und Oxidantien im Aerosol der E-Zigarette haben toxische Effekte auf das Lungenepithel. Das wirkt sich auf die Lungengesundheit junger E-Zigarettenraucher aus, wie eine US-amerikanische Studie zeigt.

Im Rahmen der Southern California Children's Health Study untersuchten die Autoren um Rob McConnell von der Abteilung für Präventivmedizin der Universität von Südkalifornien in Los Angeles Assoziationen des selbstberichteten E-Zigaretten-Konsums mit Symptomen einer chronischen Bronchitis (chronischer Husten, Auswurf oder Bronchitis) bzw. asthmaähnlichen Symptomen (Wheezing) in den vorangegangenen 12 Monaten. An der Untersuchung beteiligten sich 2086 Schüler der 11. und 12. Schuljahrgänge im Jahr 2014. 502 von ihnen (24\%) gaben an, irgendwann schon einmal eine E-Zigarette benutzt zu haben, 201 (9,6\%) waren aktuelle Nutzer, d.h. sie hatten nach eigenen Angaben innerhalb der letzten 30 Tage solche Produkte genutzt.

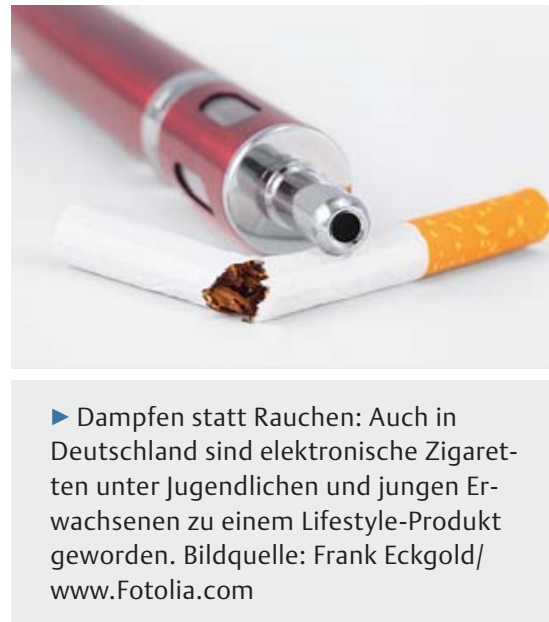

\section{Ergebnisse}

Wer angab, irgendwann in der Vergangenheit E-Zigaretten konsumiert zu haben, wies ein fast doppelt so hohes Risiko für Bronchitissymptome auf als Nie-Raucher (Odds ratio [OR] 1,85; 95\% Konfidenzintervall [KI] 1,37-2,49), aktuelle Raucher etwas über ein doppelt so hohes Risiko (OR 2,02, $95 \%$ KI 1,42-2,88). Mit der Häufigkeit des E-Zigaretten-Gebrauchs stieg das Risiko für Bronchitissymptome bei aktiven Rauchern an. Die OR im Vergleich zu Nie-Rauchern lag bei nur ein oder 2 Tagen Nutzung in den letzten 30 Tagen bei 1,66 (95\% KI 1,022,68 ), bei mindestens 3-maliger Nutzung im letzten Monat dagegen bei 2,52 (95\% KI 1,56-4,08).

Berücksichtigen die Autoren die Zahl der bisher lebenslang gerauchten Zigaretten sowie die bisherige Passivrauchexposition, schwächten sich die Assoziationen allerdings ab und waren für aktuelle E-Zi-
garetten-Nutzer nicht mehr nachzuweisen. Das erhöhte Risiko für Bronchitissymptome bei früheren E-ZigarettenRauchern blieb aber bei der Berücksichtigung solcher Einflussfaktoren bestehen und zeigte sich auch bei denjenigen, die außer einer E-Zigarette nach eigenen Angaben noch nie geraucht hatten (OR 1,70; $95 \%$ KI 1,11-2,59).

Eine Assoziation zwischen E-ZigarettenKonsum und Wheezing fand sich nicht.

FAZIT

Nach eigenen Angaben weisen adoleszente Nutzer von E-Zigaretten erhöhte Raten von Symptomen einer chronischen Bronchitis auf. Die Studie fügt der Diskussion um Sicherheit und Risiken von Zigaretten einen weiteren Puzzlestein hinzu. Die Autoren betonen, dass weitergehende Studien notwendig sind, um die Langzeiteffekte von E-Zigaretten zu untersuchen. Die FDA wird E-Zigaretten zukünftig den Tabakkontrollmaßnahmen unterwerfen, der Verkauf an Personen unter 18 Jahren wird dann verboten sein.

Friederike Klein, München 